\title{
Empowering Doctoral Candidates in Finding Relevant Concepts in a Literature Set
}

\author{
Naomi Dreher and Heinz Dreher \\ Curtin University, \\ Perth, Western Australia, Australia
}

\author{
$\underline{\text { n.dreher@curtin.edu.au, h.dreher@curtin.edu.au }}$
}

\begin{abstract}
Research activity connects prior findings with new and emerging ideas in the hope of attracting attention of those in the community who are able to make some sort of profit from the application of those ideas. Finding relevant concepts from among the vast repository of already published ideas is an important challenge for all researchers. Nowadays, there is ample opportunity to take advantage of the empowering aspects of developments in information technology. In this study, we share some ideas to efficiently and effectively find relevant concepts in a given literature set. Our need was to find and tag predetermined concepts from among the scores of research articles that our literature search had delivered - necessarily this was a large enough task to warrant the development of some automated support to find and tag the relevant concepts. We had prior exposure to Adobe ${ }^{\circledR}$ Acrobat ${ }^{\circledR}$ Pro and noted that it provides superior 'search and find' facilities; we decided to trial this use for our purposes in the 'Tribes and Cultures' research project. We have devised and trialled a seven step, semi-automated method to assist in finding relevant concepts from within collections of research articles, retrieved as Portable Document Format (PDF) files, from bibliographic databases and digital libraries.
\end{abstract}

Keywords: literature search, literature review, skim reading, concept search, concept tagging, concept mining, text mining, find and tag method, methodology.

\section{Introduction}

Searching bibliographic databases and other repositories of published literature is an essential task for researchers. This task is supported by methods developed and refined over hundreds of years. With the advent of computers and digital media, the published literature also became searchable through computerised bibliographic interfaces, initially via indexes and catalogues, as well as increasingly as full-text documents.

Non-digital forms of literature, such as reports printed on paper, require manual methods of accessing the knowledge contained therein. These methods include reading and scanning by the

Material published as part of this publication, either on-line or in print, is copyrighted by the Informing Science Institute. Permission to make digital or paper copy of part or all of these works for personal or classroom use is granted without fee provided that the copies are not made or distributed for profit or commercial advantage AND that copies 1) bear this notice in full and 2) give the full citation on the first page. It is permissible to abstract these works so long as credit is given. To copy in all other cases or to republish or to post on a server or to redistribute to lists requires specific permission and payment of a fee. Contact Publisher@InformingScience.org to request redistribution permission. human eye, photocopying portions of text, use of highlighters, sticky-notes, creating annotations, and making summaries using index cards and the like. They are labour intensive and the results of the work cannot easily be shared, stored, re-used, and managed. As more and more literature is now created, stored, and made available in digital form, researchers have the opportunity 
of revising their methods of accessing this knowledge. Such revisions can take advantage of the growing number of computer based searching and text mining techniques, knowledge management and semantic analysis approaches developed over the last few decades.

In this article, we report on the concept finding aspect of a large research project in which we needed to efficiently locate the relevant text-based concepts from within scores of research articles, and interrelate them whilst forming themes for subsequent study. Managing this process was essential to promote re-use and sharing of the concepts emerging from our literature review phase of the research. Novice researchers, including doctoral students, as well as those who are lagging in use of information technology (IT) advances may benefit from the ideas that emerged from our own intensive research. These ideas are presented herein as a case study concluding with a seven step methodology for finding relevant concepts from among hundreds of research articles.

\section{Background}

Typically, a literature review is undertaken to "gather information about a particular topic from many different but relevant sources" (Timmins \& McCabe, 2005, p. 41). The advice on how to proceed invariably mentions the need for structure, rigour, a systematic approach, and comprehensive treatment (Brereton, Kitchenham, Budgen, Turner, \& Khalil, 2007; Timmins \& McCabe, 2005). Brereton et al. (2007, Fig. 1, p. 571) presented a three phase, 10-step, "Systematic literature review process" but the entire process is manual, save for the use of search engines. A series of case studies is presented, from which they conclude that a systematic process is advisable and that existing digital libraries appear to provide better search results (in the software engineering knowledge domain) when the researcher's model of search terms conforms with that embedded in the design of the digital library. No automated support was provided for construction of the search term models.

Indeed, computer support is usually enlisted at the beginning and end of the literature review process, that is, for the bibliographic search and for reference management. This presents an opportunity to devise some digital support for the cognitive processing that takes part once the research articles have been acquired and are being used to derive meaningful information or concepts to illuminate the specific goals a researcher has in mind. Marrelli (2005), in the section "Read and Summarize the Sources" (p. 44), offered clear steps to achieve a useful outcome of the Literature Review, but all steps are entirely manual. It is left to the reader to figure out how and where some automated support may be devised and deployed.

In their article titled "The Matrix Method of Literature Reviews" by Goldman and Schmalz (2004), a stepwise advice is offered about the entire Literature Review process including the actual 'review' that requires the analysis, synthesis, and validation also mentioned by Brereton et al. (2007). These steps, we conjecture, are amenable to underpinning with IT systems.

Early in their research phase, doctoral students are confronted with finding a topic of interest and relevance to themselves and consistent with their supervisor's research interests. This topic is usually a broad area of interest and serves as an initial direction for exploration. Since a doctoral dissertation deals with specific questions or issues, the topic must be refined and distilled from the original topic area. The initial literature review serves to produce a research project of suitable scope. It must have the required degree of originality and be significant to the chosen field of study. To determine originality and significance, among other things, a thorough and comprehensive look into the existing literature is required. Clearly, a major endeavour of the doctoral candidate is reviewing the literature; empowerment via automated support for finding relevant concepts from within large quantities of research articles is the contribution of the current paper. 


\section{Automated Support for Concept Finding and Analysing}

Since technology support, as already mentioned, is well advanced for the early and final stages of the Literature Review, we set about devising a simple but powerful technology support methodology as an alternative or adjunct to skim reading; the purpose of which was to find relevant concepts in a set of retrieved research articles held in the common Portable Digital Format (PDF). We wanted our system to be fast, comprehensive, reliable, and above all, empowering to the researcher.

In their quest to find relevant articles, researchers scan bibliographic indexes and identify possible target items. For each article of possible relevance, a manual scan must be made to check if indeed the material suits the purpose. Published abstracts and annotated bibliographies assist greatly in this work because the entire article's content is reduced to a compact form. Reliance on these materials is, however, subject to their quality, and useful material can be missed due to incomplete or deficient abstracts for example. Indeed, Papaioannou, Sutton, Carroll, Booth, and Wong (2009) reported that references were missed when using what they termed 'conventional subject searching'.

Current technology permits the automatic scanning of full-text material in meaningful ways, revealing concepts of choice contained within the articles. There are a variety of methods published in the text mining literature; see for example the recent comprehensive guide titled "Text Mining: Applications and Theory" by Berry and Kogan (2010). Most of these methods are based on statistical techniques and are used in classification and clustering of textual material. The parameters for our task demanded a more specific and refined method; yet the task itself was large enough to warrant the development of some automated support to find relevant concepts. We noted that Adobe $^{\circledR}$ Acrobat ${ }^{\circledR}$ Pro provides search facilities that offer the opportunity to search for key words, select multiple instances of these words, and highlight them in a colour-coded array. Excited by the potential of these tools, we decided to investigate further by trialling its use for our purposes.

Our specific need was to find and tag predetermined concepts from among the research articles that our literature search had delivered. This literature search followed the traditional steps of creating a list of keywords, selecting relevant bibliographic databases and digital libraries, searching for articles, and then based on an initial manual scan of retrieved article title, abstract, as well as reference list (if readily available without acquiring the entire article), we made our selection for full-text article acquisition. Having obtained our initial set of articles we needed to carefully read and look for the topics or concepts of interest. As this task tends to be somewhat iterative due to concepts emerging in the researcher's mind whilst engaging with the material, as opposed to all concepts being conceived a priori, we decided to alleviate our labour by creating an alternative to skim reading.

Initially this skim reading alternative was a very methodical and manual process of creating our own synopses of each article. Later, we added the automated support method of finding and tagging relevant concepts through the use of Adobe ${ }^{\circledR}$ Acrobat ${ }^{\circledR}$ Pro. (This work was first conceptualised from our experience with semantic analysis; for example through the Automated Essay Grading project www.essaygrading.com). Tagging is needed to identify the location of concepts (represented by words or terms) within the text of the articles. This would facilitate sharing among research colleagues, analysis of ideas, and citation of literature as research reports are written.

Subsequently, we have refined and trialled a semi-automated approach to assist in finding relevant concepts in scores of research articles retrieved as PDF files from the usual literature search of bibliographic databases and digital libraries. The remainder of the present article describes the finer points of our technique, by way of a case study, and then formalises it into a methodology for finding relevant concepts that can be adopted by other researchers. 


\section{The Case Study - 'Tribes \& Cultures'}

'Tribes \& Cultures' was a research project funded by the Australian Teaching and Learning Council (ALTC), conducted in the School of Information Systems, Curtin University, Western Australia. In order to investigate issues surrounding shared professional languages and with the intent to improve learning outcomes in environments where IT and educational technology is used, we set up a project involving Educationalists who work with IT Specialists as our participants. Our hope was to determine some design guidelines and best practice methodology that could assist in the development as well as facilitation of educational software and Learning Management Systems (LMS), thus, accommodating the special needs and preferences of students studying in universities.

\section{Developing the Literature Search Criteria}

In the initial stages of the project, we developed a set of criteria (six as it eventuated) that would guide the literature review. Since we were interested in discovering ideas relating to how our key personnel groups (IT Specialists, Educationalists, \& Instructional Designers) interacted to complete their work, we made one of our criteria 'behavioural and interaction issues'; another was 'empowering learners'. From these initial ideas, explanatory statements were formed to elaborate each criterion. For 'empowering learners', the elaboration was:

Approaches/strategies for enhancing/assisting learners to acquire, understand, and create knowledge and ultimately attain higher order thinking skills. This should include the efforts made to date to ensure that developments in learning technologies are human-centred, intuitive to use, useful, and understandable.

These statements made clear our scope and intent, therefore, guiding the next step in finding relevant literature.

\section{Creating the Search Terms}

Since bibliographic databases have been constructed for traditional Boolean searching techniques, which are based on keyword lists containing inclusion and refinement logic, we needed to devise a set of search terms. This was achieved by generating terms in a series of categories that were customised to each of our six criterion statements. To ensure a comprehensive search, it is necessary to not only carefully select the relevant bibliographic databases and digital libraries, but also to phrase search terms with care. Our first step was to develop what we have termed the key phrases. This was achieved by deconstructing and analysing the explanatory statements for each criterion.

To begin the process, each criterion's key phrases were deconstructed into key terms containing the specific terms that broadly encompassed the criterion and would theoretically extract the largest portion of relevant documents from the broad literature base. Expanding upon this, several themed sub-categories were drawn from each of the criterion statements and important key terms were listed beneath each sub-category. Such an approach was designed to uncover the more specialised literature articles, which may only in part be relevant to the criterion.

Necessarily, to ensure complete literature retrieval, a variety of alternative constructions of the key phrases were required. For example, "higher order thinking skills" was developed into five key phrases: high level thinking, higher order, higher order learning, higher order thinking, and thinking skills (see Table 1). Our experience with initial searching has shown that these are likely to deliver better results for our purposes than just the one long phrase from the explanatory statement. 
Individual word forms needed consideration too. For example, whilst the terms representing our search concepts were "cognitive", "creation", and "thinking" (see the "Assisting Learners' category in Table 1) only the highlighted portion, or word stem, was used as the search term; thus "cogniti" rather than "cognitive" as this would result in targeting "cognition" as well, but not "cognize" or "cognate". Of course, using such techniques is an approximation and what one really wants is a conceptual searching environment such as is embedded in the MarkIT Automated Essay Grading System (Dreher, 2006; Williams, 2006).

It is important to note that these lists were not created in one work session, but rather emerged after some considerable experimentation. This method of search term refinement produced the search terms by category for each of the six criteria that guided our literature review. Once these terms had been used to identify our target research papers, 68 in all, they were further used in finding the relevant concepts within these target documents as explained in a subsequent section: Keyword finding and tagging.

The search term refinement method for the criterion "Approaches/strategies for enhancing/assisting learners to acquire, understand, and create knowledge and ultimately attain higher order thinking skills" produced three categories: 'Key Phrases', 'Assisting Learners', and 'Learning Technology Development'.

Table 1: Categories of Search Terms

\begin{tabular}{|c|l|l|}
\hline Key Phrases & Assisting Learners & Learning Technology Development \\
\hline cognitive skills & assisted learning & developments \\
\hline enhanced learning & cognitive & educational \\
\hline enhancing learning & creation & educational application \\
\hline high level thinking & enhance & educational courseware \\
\hline higher order & intelligence & educational hypermedia \\
\hline higher order learning & knowledge & educational media \\
\hline higher order thinking & learning environments & educational multimedia \\
\hline thinking skills & thinking & educational software \\
\hline & understanding & educational system \\
\hline & & educational technology \\
\hline & & human centered \\
\hline & & human centred \\
\hline & & intuition \\
\hline & & learning system \\
\hline & & learning technology \\
\hline & & online courseware \\
\hline & & useful \\
\hline
\end{tabular}

The search terms within the categories were colour coded for two separate reasons - firstly to indicate the particular theme of each search term. In Table 1, the yellow terms relate to educational theory, whilst the blue terms relate to the supporting educational technologies. Secondly, colour coding was used to indicate search term truncation for Boolean search purposes.

\section{Using the Search Terms in Bibliographic Search}

Using our search terms, developed from the literature search criteria, we used the bibliographic database search interface Gecko, as provided by our library, and targeted an appropriate selection of full-text article databases (explaining the choice of which is itself a non-trivial task and is outside the scope of the present article). Our search delivered a large selection of articles that was quickly refined to a set of 68 ; the process of which will now be explained. 
The literature search was conducted in two phases; the initial phase made use of the key phrases developed for each criterion. The key phrases category (see Table 1, first column) denotes which specific terms were used to broadly encompass the criterion and extract relevant documents from the bibliographic databases. Specific documents in the broad literature base needed to contain multiple instances of two or more of the key terms in order to qualify in the short-list of literature for each of our six criteria. Bibliographic database searches were conducted in the usual manner of using strings of phrases, separated by commas.

From the resulting set of articles the lists of key phrases were further refined into the customised categories for each criterion and a secondary literature search was conducted. The subsequent search made use of the university library "Gecko" search system as well as Google Scholar. Each database returned many similar but also different results; however, one obvious advantage to using Gecko, which has active subscriptions to a vast array of electronic journals, is that from there one can easily obtain full-text PDF documents via a single hyperlink. This is not so easy with Google Scholar, where in many cases one must then perform an additional search of the specific article name, from within a subscribed database, in order to find a full-text PDF.

\section{Organising and Managing the Retrieved Articles}

Typically, the filenames of articles downloaded from literature repositories are meaningless in the individual research context. After much experimentation over the years we have found it best to include some identifying 'tags' or text strings within the filename, which represent author name, publication year, and article title. The challenge is to provide enough meaning within a relatively short string of characters. Thankfully we are not restricted to the eight character filenames of the DOS days! An example of an article from our case study reference list is "Berry, M. W., \& Kogan, J. (Eds.). (2010). Text Mining: Applications and Theory", which would have the corresponding filename of "Berry\&Kogan(Eds)_TextMining(2010)". Sometimes the underscore " " charac-

ter is used in place of parentheses, and the year may appear directly after the authors, however the author names always appear first and if there were many authors then we would use "_etal" after the first author name.

We endeavoured to obtain PDF versions of all articles, which is somewhat of a standard now; however at times there are many Microsoft Word (.doc or .docx) versions available, especially of documents we create ourselves. A good example is the article synopsis that we created for each research article that we finally decide to use (a process to be explained below). For such cases we have devised a workflow using the "Automator" application on the Apple Macintosh, which creates a PDF for each Microsoft Word formatted file in a selected list. In any event, our goal of finding relevant concepts in the acquired research articles is dependent on Adobe ${ }^{\circledR}$ Acrobat ${ }^{\circledR}$ Pro PDF objects.

Once we had standardised and settled on our filenames for the research articles, we arranged them into a suitable directory structure. In the current case study it is apparent that one structure could be based on the six criteria mentioned. The problem with this approach is that an article may pertain to more than one criterion, and naturally we do not want to store redundant copies of the material. Actually, one can readily appreciate here that which the database designers have wrestled with for the entire time they insisted on having hierarchical structures. Hypertext technology has shown us that one can have the objects in one physical place, as it were, and create lists of pointers to provide the multiple views needed for the research project. An example of the use of these in practice is Google's Gmail, where items are tagged, or pointed to via a 'tag'. An item may have many tags, and many items may have the same tag. There is no concept of hierarchy at all it is pure hypertext in action. Readers may compare this with the traditional approach of storing mail items in 'folders' and reflect on the relative merits of each system. Our reflections have resulted in a linear list of research article objects. Although, we should say that where subsequent 
searches have revealed new or further articles these are stored in a new folder so that we have the literature search results in various stages of treatment physically separated. Once our project was nearing completion we combined all our retrieved articles into one folder containing the PDF version and another containing the Microsoft's Word (.doc or .docx) version (note: any pure text material is stored in Microsoft's Word file format). Finally, since we usually work in teams, the members of which are dispersed in time or place, we used a cloud-computing device (for example DropBox or Skype) to share these artefacts.

\section{Keyword Finding and Tagging}

Thus far we have invested considerable resources in acquiring literature that, by way of our search techniques, is likely to have a very high level of relevancy (68 articles in our case), whilst minimising instances of largely irrelevant articles filtering in. The benefits of these efforts become evident when the researcher begins the initial process of making practical use of the material - that is, by generating ideas, following conceptual threads, linking to text fragments, quoting parts of the literature, and revisiting specific parts of the material. All these activities are based on a very usable 'Search' function in Adobe ${ }^{\circledR}$ Acrobat $^{\circledR}$ Pro - one whose invocation and operation remains in the background, therefore allowing the researcher to concentrate on the emerging ideas.

As we process the research material in this way, we build 'views' or conceptual structures (networks of ideas \& concepts). Our concept-views would be used as the basis for connecting emerging ideas from research field data and the extant published research articles. However, these concept-views are created on-the-fly and, thus, need to be preserved in some way for re-use by colleagues or subsequently. Thus, we needed to devise a method for creating the concept-views, which provided a framework for use and re-use. We began with our search terms list created during the literature databases searches; the structured list from Table 1 was 'unpacked' into words, 'linearised', and ordered alphabetically to arrive at Figure 1: Relevant Key Terms.

Highlighting has been used to indicate search word truncation in order to achieve maximum coverage of the various available forms of key terms. The key terms that proved the most valuable for finding relevant literature have been highlighted in green, all other terms are yellow. 


\title{
Relevant Key Terms
}

\author{
(a short list)
}

This alphabetical list of key terms can be used to find additional literature (if necessary) for the Tribes and Cultures project.

Highlighting has been used to indicate the relevant truncated section of each word.

Terms highlighted in green hold the highest relevancy. Terms highlighted in yellow hold secondary importance.

NOTE: See the 'Search_Methods' document for additional information on conducting a literature review search for the Tribes and Cultures project.

$\begin{array}{llll}\text { application } & \text { domain } & \text { intuition } & \text { prospective } \\ \text { approaches } & \text { earning } & \text { IT tech } & \text { quality } \\ \text { assess } & \text { economical } & \text { knowledge } & \text { relation } \\ \text { assisted } & \text { educational } & \text { language } & \text { results } \\ \text { authoring } & \text { educationalists } & \text { learn } & \text { saving } \\ \text { behaviourism } & \text { educators } & \text { learner } & \text { semantic } \\ \text { bodies } & \text { effective } & \text { learning } & \text { sharing } \\ \text { budget } & \text { effectiveness } & \text { lecturer } & \text { skills } \\ \text { centered } & \text { efficiency } & \text { level } & \text { software } \\ \text { centred } & \text { enhanced } & \text { low cost } & \text { solutions } \\ \text { cognitive } & \text { environments } & \text { matter } & \text { specialists } \\ \text { collaboration } & \text { evaluation } & \text { meaningful } & \text { strategies } \\ \text { collaborative } & \text { expectations } & \text { media } & \text { student } \\ \text { collegial } & \text { experts } & \text { mentor } & \text { subject } \\ \text { communication } & \text { funds } & \text { methodologies } & \text { system } \\ \text { community } & \text { gap } & \text { money } & \text { teacher } \\ \text { computer } & \text { goals } & \text { multidisciplinary } & \text { team } \\ \text { constructivism } & \text { guidelines } & \text { multimedia } & \text { tech } \\ \text { cooperation } & \text { high } & \text { online } & \text { technician } \\ \text { cost } & \text { higher } & \text { ontology } & \text { technology } \\ \text { courseware } & \text { human } & \text { order } & \text { terminology } \\ \text { creation } & \text { hypermedia } & \text { outcome } & \text { theory } \\ \text { culture } & \text { imperatives } & \text { outcomes } & \text { thinking } \\ \text { design } & \text { implementation } & \text { pedagogy } & \text { tutor } \\ \text { determine } & \text { improved } & \text { performance } & \text { understanding } \\ \text { develop } & \text { improvements } & \text { possibilities } & \text { usability } \\ \text { developer } & \text { Information } & \text { potentialities } & \text { useful } \\ \text { developments } & \text { instructional } & \text { principle } & \text { vocabulary } \\ \text { dialogue } & \text { intelligence } & \text { problem } & \\ \text { digital } & \text { interaction } & \text { professionals } & \\ & & & \end{array}$

\section{Figure 1: Relevant Key Terms}

Depending on what concept-view we wanted to generate, we made a selection of key-terms and a selection of target research articles (from among our 68 documents, all in PDF format) and used the Adobe ${ }^{\circledR}$ Acrobat ${ }^{\circledR}$ Pro "Search" function. In our example we were interested in the "evaluating learning systems" concept comprising student performance, quality outcomes, and including methodological aspects. The appropriate key-terms were selected from Figure 1. 
For our criterion:

Evaluation guidelines in use that assist to determine the quality of learning outcomes in relation to electronic education software

Two categories of terms were decided upon: those pertaining to 'evaluation'; and, those directly mentioning learning systems (see Table 2: Specific Categories of Search Terms). When a different highlight colour was assigned to each category, upon completion of the word search it would become quickly evident via the dispersion of coloured highlights which 'evaluation' terms related to 'learning systems' and which did not - thus, minimising the volume of further reading necessary. Additionally, a high degree of highlighted text would indicate how relevant an entire article was to each of our separate criteria.

Table 2: Specific Categories of Search Terms

\begin{tabular}{|l|l|}
\hline Evaluation Concepts & Learning System Concepts \\
\hline evaluat & learn \\
\hline student & system \\
\hline perform & software \\
\hline outcome & management \\
\hline method & educat \\
\hline & environment \\
\hline
\end{tabular}

To perform such a search, the 'Search' function of Adobe ${ }^{\circledR}$ Acrobat ${ }^{\circledR}$ Pro is used; it is found in the Edit menu. The method we followed is universal to Mac and PC platforms in the most recent editions of Adobe ${ }^{\circledR}$ Acrobat $^{\circledR}$ Pro - we were using versions 8 and 9. With the search window open, at the bottom of the window we invoked 'Use Advance Search Options'. The method of searching described here does not function in the 'Basic Search Options'. With the Advanced Search Options displayed, we entered our truncated key terms (separated by a space; do not separate by commas) into the 'What word or phrase would you like to search for?' dialogue box. In the 'Result results containing:' drop-down menu, we chose 'Match Any of the words'. Then in the 'Look In:' drop down menu one can specify to target the search in 'The Current PDF Document'; alternatively, through 'Browsing for a location' and then selecting the containing folder, it is possible to target a collection of documents that are stored on an accessible storage device. From the check box list of 'Use these additional criteria:' ensure that the 'Whole words only', 'Case-Sensitive' and 'Stemming' check boxes are unchecked. This may seem curious as we are clearly using words stems in our search; however, we wanted full control of all the word stems and word forms to be used. Figure 2: Searching for the "Evaluating Learning" Concept, depicts the dialogue box entries for the example search just described.

Search results are displayed as a list of instances of each word found in the specified document/s. In Figure 3: Search Results From the "Evaluating Learning" Concept, we see 204 instances as found in the one article "Ardito et al." of concepts that relate to our 'evaluation' category depicted in Table 2. 


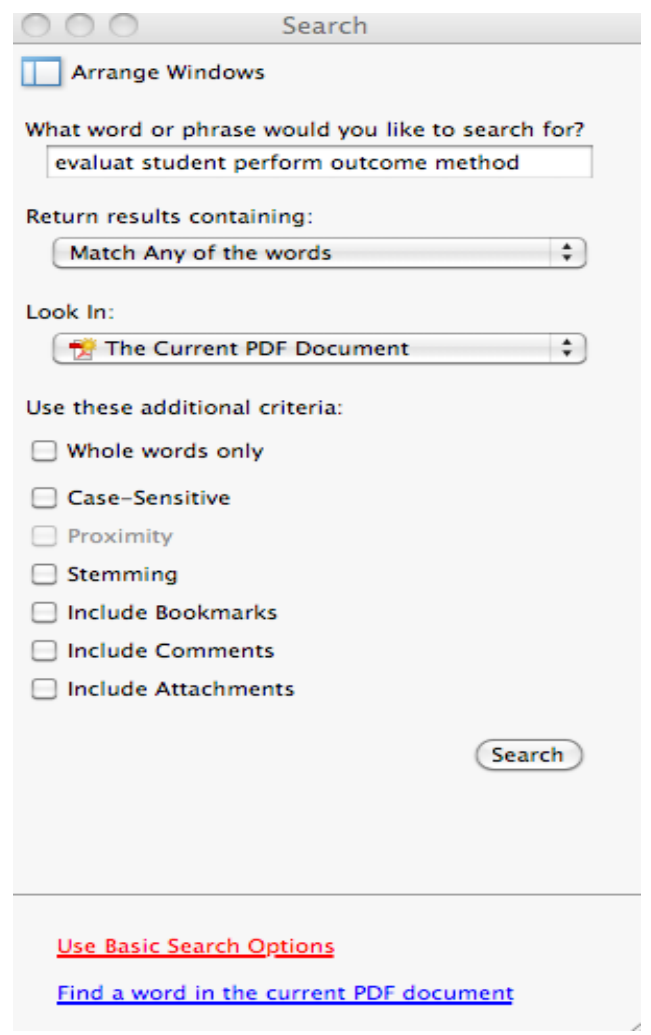

Figure 2: Searching for the "Evaluating Learning" Concept

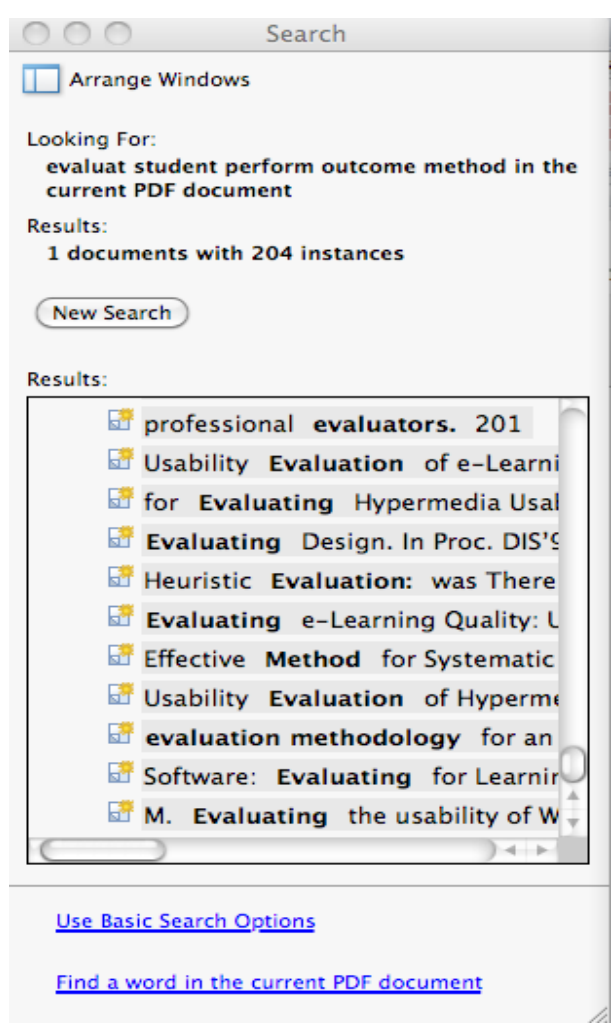

Figure 3: Search Results From the "Evaluating Learning" Concept 
Specific instances of terms can be selected from the search results list, which will then be displayed in the document. Alternatively, all instances from the search results list can be selected in the document at once by selecting the first term in the search results list and then 'shift clicking' on the last term. To then apply a highlighting colour to these terms in the document, one must first ensure the 'Select' tool is active then right click on one of the highlighted instances in the document and lastly click on 'Highlight Text (Comment)' from the pop-up menu. This will highlight the text with the default colour (yellow) or the last colour specified by the user. When performing a multi-document search, this same processing of applying coloured highlighting holds true; however, each document must be processed individually. That is to say, when a document title is clicked on in the list of search results, which may contain 12 documents to choose an arbitrary number, this document will then open. Next the user must select the appropriate search terms from this list and follow the highlighting process 12 times in total to process all documents in the multi-document search. This process could be automated, at least in part, via macro or workflow technology.

When performing additional searches that necessitate highlights to be made in a different colour it is necessary to first change the default highlight colour before commencing the search. With the 'Comment and Markup' toolbar turned on (available from the View > Toolbars menu), one can then right click on the 'Highlight Text' tool button from the menu bar and choose 'Default Tool Properties' from the pop-up menu that appears. From here the default highlight colour can be changed. The first page of one research article, with highlighted key-terms is depicted in Figure 4.

This output of our "Finding Relevant Concepts" methodology may be shared among researchers and further refined to suit the purpose. In the absence of using such a method, the researcher would do something equivalent but 'all in the mind' as it were, and there would be no consistent replicable method, no sharable outcome, and no preserved artefact to persist in the research record.

Having now created one or more concept-views of the already acquired literature set we could make fine grained judgement about these articles, such as whether they were indeed suitable for our purpose, and could then be included in the next, rather labour intensive stage of the overall literature review process - that of creating article synopses. 


\title{
Systematic Evaluation of e-Learning Systems: An Experimental Validation
}

\author{
C. Ardito ${ }^{\circ}$ M. F. Costabile ${ }^{\circ}$, A. De Angeli*, R. Lanzilotti ${ }^{\circ}$ \\ 'Dipartimento di Informatica, Università di Bari, Italy \\ \{ardito, costabile, lanzilotti\}@di.uniba.it \\ "School of Informatics - University of Manchester Po BOX 88 M601QD - UK \\ Antonella.de-angeli@manchester.ac.uk
}

\begin{abstract}
The evaluation of e-learning applications deserves special attention and evaluators need effective methodologies and appropriate guidelines to perform their task. We have proposed a methodology, called eLSE (e-Learning Systematic Evaluation), which combines a specific inspection technique with user-testing. This inspection aims at allowing inspectors that may not have a wide experience in evaluating e-learning systems to perform accurate evaluations. It is based on the use of evaluation patterns, called Abstract Tasks (ATs), which precisely describe the activities to be performed during inspection. For this reason, it is called AT inspection. In this paper, we present an empirical validation of the AT inspection technique: three groups of novice inspectors evaluated a commercial elearning system applying the AT inspection, the heuristic inspection, or user-testing. Results have shown an advantage of the AT inspection over the other two usability evaluation methods, demonstrating that Abstract Tasks are effective and efficient tools to drive evaluators and improve their performance. Important methodological considerations on the reliability of usability evaluation techniques are discussed.
\end{abstract}

\section{Author Keywords \\ E-learning system evaluation, usability evaluation techniques, controlled experiment.}

\section{ACM Classification Keywords}

H5.m. Information interfaces and presentation (e.g., HCI): Miscellaneous.

\section{INTRODUCTION}

E-learning is the most recent way to carry out distance

Permission to make digital or hard copies of all or part of this work for personal or classroom use is granted without fee provided that copies are not made or distributed for profit or commercial advantage, and that copies bear this notice and the full citation on the first page. To copy otherwise, to republish, to post on servers or to redistribute to lists, requires prior specific permission and/or a fee.

NordiCHI 2006: Changing Roles, 14-18 October 2006, Oslo, Norway

Copyright 2006 ACM ISBN 1-59593-325-5/06/0010 ..\$5.00 education by distributing learning material and processes over the Internet. Its "any time, any place" nature could be part of a winning strategy for particular needs, such as decongestion of overcrowded education facilities, support for students or teachers who live far away from schools and universities, and adult education.

Making remote educational data and tools available to learners requires considering their different characteristics, such as cultural background, technical experience, technological equipment, and physical/cognitive abilities. Thus, a major challenge for designers and HumanComputer Interaction (HCI) researchers is to develop software tools to engage novice learners and support their learning even at a distance. This could require revising traditional interaction paradigms to provide new flexibility and adaptiveness, to account for the peculiarities of the specific application field. Towards this end, there should be a synergy between the learning process and the student's interaction with the software. As for any interactive system, the quality of the user interface is a primary requirement. If the e-learning system interface is not usable, the student could spend more time learning how to use the system rather than learning the educational content. Thus, in this particular context, issues of usability will assume greater significance. Besides being usable, an e-learning system must be effective in meeting the instructor's pedagogical objectives and the student's needs. Interface elements and functionalities, information quality and pedagogical content should support people to learn in various contexts according to selected pedagogical objectives. As a consequence usability evaluation need to integrate the assessment of pedagogical quality of e-learning.

For the above reasons, the evaluation of e-learning systems deserves special attention, and evaluators need appropriate guidelines as well as effective evaluation methodologies 15]. Unfortunately, the number of studies addressing usability of e-learning systems is relatively small and inadequate to the importance of the issue $[11,13]$. Moreover, it is often the case that the evaluation criteria are only vaguely stated $10,12,14]$, so that an actual measurement of the system quality is left to subjective interpretation.

Figure 4: The "Ardito et al." article with highlighted key-terms 


\title{
Creating Article Synopses
}

When an article has been identified as being useful, by using all search and find techniques that are suitable, including the keyword finding and tagging approach explained, we invest in creating an article synopsis. We have developed a standard framework or template for this, as shown in Figure 5: Article Synopsis Template.

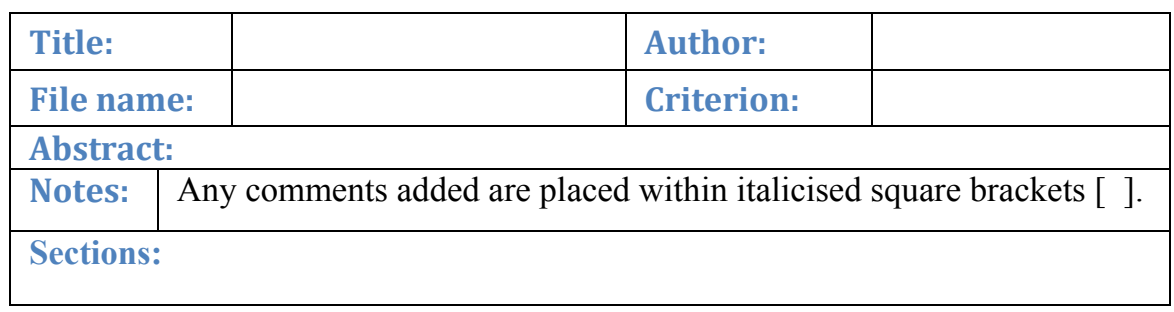

Figure 5: Article Synopsis Template

After creating many synopses, a more or less standard process or workflow has emerged. It is a guide, and we cannot always adhere to it, especially the one-page-per-research-article restriction. The Synopsis Process is depicted in Figure 6: The Synopsis Creation Workflow.

\section{Synopsis Process}

\author{
No more than one page per paper \\ Include: \\ - Title of paper \\ - Author \\ - File name \\ - All criterion relevant \\ - Abstract \\ - $1^{\text {st }}$ and last sentence of each heading \\ - Glean $\mathrm{b} / \mathrm{t}$ for anything more particularly relevant \\ Key Themes (purpose for synopsis): \\ 1. What not to do! \\ 2. What is relevant! \\ Identifying the key papers for developing a suitable methodology for the project.
}

\section{Figure 6: The Synopsis Creation Workflow}

Obviously, the elements within the template can be varied according to specific requirements. From Figure 6 it can be seen that this template is akin to a set of prompts for a particular researcher to be guided by, the result of which can be seen in Figure 7: The "Ardito et al." Synopsis. 


\begin{tabular}{|l|l|l|l|}
\hline Title: & $\begin{array}{l}\text { Systematic Evaluation of e-Learning Systems: An } \\
\text { Experimental Validation }\end{array}$ & Author/s: & $\begin{array}{l}\text { C. Ardito, M. F.; Costabile, A. } \\
\text { De; Angeli, R.; Lanzilotti, S. }\end{array}$ \\
\hline File name: & $\begin{array}{l}\text { Ardito (2006) Systematic evaluation of eLearning } \\
\text { systems }\end{array}$ & Criterion: & $2,3,4,6$ \\
\hline $\begin{array}{l}\text { Abstract: } \\
\text { The evaluation of e-learning applications deserves special attention and evaluators need effective methodologies and } \\
\text { appropriate guidelines to perform their task. We have proposed a methodology, called eLSE (e-Learning Systematic } \\
\text { Evaluation), which combines a specific inspection technique with user-testing. This inspection aims at allowing inspectors that } \\
\text { may not have a wide experience in evaluating e-learning systems to perform accurate evaluations. It is based on the use of } \\
\text { evaluation patterns, called Abstract Tasks (ATs), which precisely describe the activities to be performed during inspection. For } \\
\text { this reason, it is called AT inspection. In this paper, we present an empirical validation of the AT inspection technique: three } \\
\text { groups of novice inspectors evaluated a commercial eLearning system applying the AT inspection, the heuristic inspection, or } \\
\text { user-testing. Results have shown an advantage of the AT inspection over the other two usability evaluation methods, } \\
\text { demonstrating that Abstract Tasks are effective and efficient tools to drive evaluators and improve their performance. } \\
\text { Important methodological considerations on the reliability of usability evaluation techniques are discussed. }\end{array}$ \\
\hline \\
Notes: $\quad$ Any comments added are placed within italicised square brackets [ ]. \\
\hline
\end{tabular}

Sections:

\section{INTRODUCTION}

eLearning is the most recent way to carry out distance education by distributing learning material and processes over the Internet.

The paper has the following organization. Next section briefly illustrates the basic usability evaluation techniques and provides the rationale for the AT inspection, which is described in the successive. The section "The Experiment" reports the validation experiment and the section "Conclusion" closes the paper.

\section{USABILITY TECHNIQUES}

Different methods can be used for evaluating the usability of interactive systems. Among them, the most commonly adopted are user-based methods and inspection methods

In this paper, an empirical evaluation of the AT inspection to evaluate e-learning systems is presented.

\section{AT INSPECTION}

According to the eLSE methodology, the AT inspection is the basic activity to perform. The eLSE methodology was proposed to make the usability evaluation of e-learning systems more systematic, efficient, and reliable. [Characteristics of the eLSE methodology are explained.]

Usability criteria and ATs for the platform have been defined that differ from that defined for e-learning modules, since different features and criteria need to be considered in these two contexts.

\section{THE EXPERIMENT}

In order to validate the AT inspection technique, a comparison study involving 73 senior students of a $\mathrm{HCl}$ class at the University of Bari in Italy has been conducted. The aim of the experiment was to compare the performance of evaluators carrying out the AT inspection with the performance of evaluators carrying out a heuristic inspection, or conducting traditional user testing.

In the following sections we describe the experimental method adopted to test these hypotheses [these being 'effectiveness', 'efficiency' and 'satisfaction' - method, results and a discussion are presented].

\section{CONCLUSIONS}

One present goal of Human-Computer Interaction researchers and developers is to create software tools that support people to learn in an effective manner the material available online.

To better assess the value of the AT inspection, we plan to conduct in the future a similar experiment involving professional evaluators.

\section{Figure 7: The "Ardito et al." Synopsis}


Creating the synopses followed a fairly structured and straightforward, yet nevertheless time consuming process - there are perhaps opportunities for automation here. The synopses were created one by one for each of the 68 articles that were found to be most relevant based upon the key-term searching accomplished via Adobe ${ }^{\circledR}$ Acrobat ${ }^{\circledR}$ Pro. Firstly the relevant identifying information was entered into the synopsis template; that is, the title of the paper, the author, and the filename. Following this, all relevant criteria (these were established during the key-term search of the articles and stored in a spreadsheet resembling an annotated bibliography) were listed, and the abstract of the article was pasted into the synopsis template.

Next, came the time consuming task of listing all major headings from the article in order of appearance; the first and last sentence of the content therein was copied or paraphrased, this proving insufficient, occasionally more information was skim read and added. One can readily automate part of this in the future. The synopses were generated with the intent of identifying key concepts that would assist in refining our methodology for the project by way of identifying relevant topics as well as those areas to avoid - relevant concepts, and non-relevant concepts. This was most useful for those researchers in the team who had not been involved in the process of searching for and tagging the concepts, as it provided a quick and efficient way to establish an overview of the literature that had been acquired. A meta-analysis could also be performed on the literature set by applying the "keyword finding and tagging" to the synopses themselves.

Whether using the synopses or the research articles themselves, the keyword finding and tagging is an essentially automatic process supported by a fully functional search facility such as that found in Adobe ${ }^{\circledR}$ Acrobat $^{\circledR}$ Pro, its outcome being an all important conceptual view available via clusters of embedded concepts highlighted in situ and in context within and across the research articles - we call these concept-views.

\section{Conclusion - "Finding Relevant Concepts Method"}

In the preceding sections we have outlined and exemplified a series of seven steps which is intended to methodologise the tasks associated with finding relevant concepts from among the hundreds of research articles, with their thousands of pages, and hundreds of thousands of words; enabling researchers to obtain benefit from information technologies now readily available, and thereby free their cognitive resources for doing the truly creative work needed in all research projects. Table 3 provides a summary (with outcomes) of the seven steps in our 'Finding relevant concepts method'.

Table 3: Finding Relevant Concepts - Seven Steps

\begin{tabular}{|l|l|}
\hline Finding relevant concepts - seven steps & Outcomes of each of the seven steps \\
\hline Develop the literature search criteria & $\begin{array}{l}\text { initial ideas; explanatory statements; search } \\
\text { criteria }\end{array}$ \\
\hline Creating the search terms & $\begin{array}{l}\text { deconstructed explanatory statements; hierar- } \\
\text { chies and lists of key phrases and search } \\
\text { terms; categories of search terms }\end{array}$ \\
\hline Using the search terms in bibliographic search & potential set of relevant research articles \\
\hline Organising and managing the retrieved articles & $\begin{array}{l}\text { an organised, usable, and shareable set of rele- } \\
\text { vant research articles }\end{array}$ \\
\hline Keyword finding and tagging & $\begin{array}{l}\text { relevant key terms; research articles and syn- } \\
\text { opses with embedded highlighted key-terms }\end{array}$ \\
\hline Creating article synopses & $\begin{array}{l}\text { one page research article synopses focussed on } \\
\text { research project literature search criteria }\end{array}$ \\
\hline Generating literature concept overviews & $\begin{array}{l}\text { found relevant concepts; concept-views; high- } \\
\text { lighted terms within articles }\end{array}$ \\
\hline
\end{tabular}




\section{Future Developments}

As has been noted by Brereton et al. (2007), the domain specific idiosyncrasies of some digital libraries makes literature searching a less satisfying and more arduous task than is desirable. It may be worth investigating the possibility of interoperating the "Finding relevant concepts seven steps" methodology with digital libraries on a case-by-case basis. This may, for example, be accomplished by creating abstract models of search terms for specific digital libraries, and defining an interface between these and our seven step model.

\section{Acknowledgements}

Support for the 'Tribes \& Cultures' project referred to in this article has been provided by the Australian Learning and Teaching Council Ltd, an initiative of the Australian Government Department of Education, Employment and Workplace Relations. The views expressed in the project do not necessarily reflect the views of the Australian Learning and Teaching Council Ltd.

\section{References}

Ardito, C., Costabile, M. F., De Angeli, A., \& Lanzilotti, R. (2006). Systematic evaluation of e-learning systems: An experimental validation. NordiCHI 2006: Changing Roles, 14-18 October 2006, Oslo, Norway."

Berry, M. W., \& Kogan, J. (Eds.). (2010). Text mining: Applications and theory. Wiley, Chichester, UK. Retrieved November 21, 2010 from http://www.ebook3000.com/Text-Mining--Applications-andTheory 56117.html

Brereton, P., Kitchenham, B. A., Budgen, D., Turner, M., \& Khalil, M. (2007). Lessons from applying the systematic literature review process within the software engineering domain. The Journal of Systems and Software, 80, 571-583.

Dreher, H. (2006). Interactive on-line formative evaluation of student assignments. In Proceedings of InSITE 2006, June 25-28, Salford (Greater Manchester, England). Retrieved February 28, 2011, from http://proceedings.informingscience.org/InSITE2006/IISITDreh235.pdf

Goldman, K. D., \& Schmalz, K. J. (2004). The matrix method of literature reviews. Health Promotion Practice. Sage Publication: Thousand Oaks, CA. Retrieved: February 28, 2011, from http:/hpp.sagepub.com/content $/ 5 / 1 / 5$.citation

Marrelli, A. F. (2005). The performance technologist's toolbox: Literature reviews. Performance Improvement, 44, 7. ABI/INFORM Global.

Papaioannou, D., Sutton, A., Carroll, C., Booth, A., \& Wong, R. (2009). Literature searching for social science systematic reviews: Consideration of a range of search techniques. Health Information and Libraries Journal, 27, 114-122. DOI: 10.1111/j.1471-1842.2009.00863.x

Timmins, F., \& McCabe, C. (2005). How to conduct an effective literature search. Nursing Standard, 20(11), 41-47.

Williams, R. (2006). The power of normalised word vectors for automatically grading essays. In Proceedings of InSITE 2006, June 25-28.Salford (Greater Manchester, England). Retrieved February 28, 2011, from http://proceedings.informingscience.org/InSITE2006/IISITWill155.pdf 


\section{Biographies}

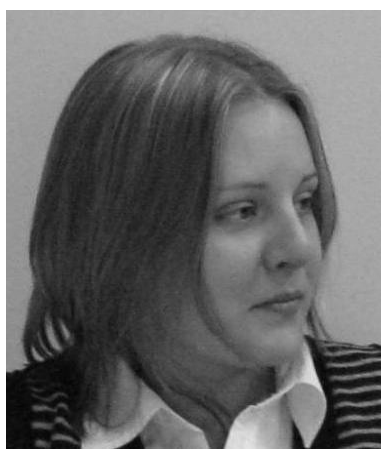

Naomi Dreher is a Research Associate for the Semantic Analysis and Text Mining for Business and Education (SATM4BE) research group at Curtin University, Perth, Western Australia. She also teaches Information Technology at Cyril Jackson Senior Campus, a Perth secondary school for mature aged and refugee students. In her undergraduate studies Naomi completed a Bachelor of Communications with Honours; she also has a Certificate IV in Information Technology, a Diploma of Information Technology; a Graduate Diploma of Secondary Education; and a Certificate IV in Training and Assessment. As an early career researcher, Naomi is focused on developing her career as an educator and further researching in the field of computer assisted

education and assessment.

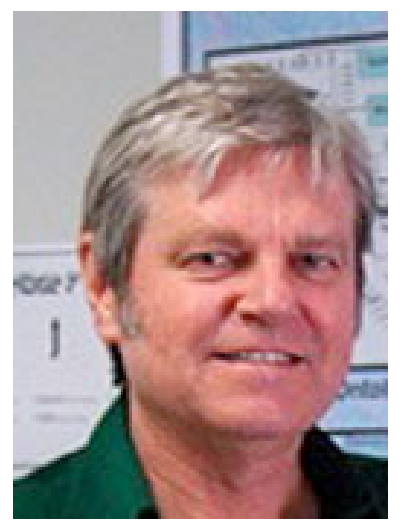

Heinz Dreher is a Professor in Informatics at the Curtin Business School, Curtin University, Perth, Western Australia. He has published in the educational technology and information systems domain through conferences, journals, invited talks and seminars; is currently the holder of Australian National Competitive Grant funding for a 4-year E-Learning project and a 4-year project on Automated Essay Grading technology development, trial usage and evaluation; has received numerous industry grants for investigating hypertext based systems in training and business scenarios; and is an experienced and accomplished teacher, receiving awards for his work in cross-cultural awareness and course design. In 2004 he was appointed Adjunct Professor for Computer Science at TU Graz, Austria, and continues to collaborate in teaching \& learning and research projects with European partners. Dr Dreher's research and development programme is supported by Curtin Business School Area of Research Focus funding - Semantic Analysis and Text Mining for Business and Education (www.eaglesemantics.com) in addition to other competitive funding obtained for individual projects. 